\title{
QUELQUES ASPECTS DE L'ART MILITAIRE SOUS PHILIPPE AUGUSTE
}

\author{
PAR \\ J.-F. FI NO
}

LE règne de Philippe Auguste est marqué par le long conflit qui l'oppose aux Plantagenêts. C'est une lutte de plus de trente ans, jalonnée tantôt par les défaites du Capétien (Fréteval 1194, Courcelles 1198), tantôt par ses victoires diplomatiques ou militaires aboutissant à la conquête de places telles Gisors, Château-Gaillard, Loches, Chinon. Finalement, les Plantagenêts sont vaincus et l'annexion de la Normandie, de la Touraine, de l'Anjou, de l'Artois, de l'Ammiénois, de l'Auvergne et d'une partie du Poitou, font du roi de France le plus puissant souverain d'Europe.

Cette lutte a de profondes répercussions sur l'évolution de l'art militaire et il est peut-être opportun de rappeler quelques uns des travaux qui ont été consacrés à trois aspects fondamentaux: les armes, les effectifs, les bâtiments.

\section{LES ARMES}

Au cours de ces guerres continuelles, l'armement ne cesse de se perfectionner ${ }^{1}$. Il est vrai que fort peu d'armes de cette époque sont parvenues jusqu'à nous. On est beaucoup mieux renseigné sur celles appartenant à la période franque ${ }^{2}$ car, déposées dans les sépultures à côté du

'E. Viollet-Le-Duc: Dictionnaire raisonné du mobilier français de l'époque carlovingienne à la Renaissance, Paris, Bance, 1858-1875, 6 vols.; cf. Outils, outillages, vol. 2, $6^{*}$ partie; Armes de guerre, offensives et défensives, vols. 5 et 6 . E. Vio LLIT-LE-DuC: Dictionnaire raisonné de l'architecture française du XI au XVI siècles, Paris, Bance, 1858-1868, 10 vols. H. SABINE: Table analytique et synthètique du. dictionnaire raisonné de l'architecture française du XI au XVI siècles, Paris, Libr. des Imp. Réunies, 1889 , XX, 387 p. Ces Dictionnaires ont vieillis, certaines illustrations ne sont que des interprétations, mais ils n'ont pas été remplacés. D’utiles indications pourront être trouvées in: C. ENLART: Manuel d'archéologie française depuis les temps mérovingiens jusqu'à la Renaissance, Paris, Picard, 1902-1916, 3 vols. (Le costume, vol. 3); W. Boенеim: Handbuch der Waffenkunde... von Beginn des Mittelalters bis zum Ende des 18. J., Leipzig, E. A. Seeman, 1890, VIII, 495 p. (Réimpr. Gratz, Akademische Druck u. Verlag, 1966).

${ }_{2}$ La terminologie traditionnelle est sujette à caution: «Le seule arme employée par les Barbares dont on puisse connaître le nom qu'elle portait au temps des grandes Invasions est probablement l'angon... Pour les autres, il est préférable de parler 
défunt, un certain nombre nous ont été livrées par les fouilles ${ }^{3}$. Par contre, l'usage d'enterrer le guerrier avec ses armes ayant été prohibé par Charlemagne et la pénurie de fer, chronique au Moyen Age, ayant été cause que la plupart des objects brisés ou hors d'usage aient été refondus pour en récupérer le métal, les collections des musées sont extrèmement pauvres à ce point de vue. Force est donc de recourir aux représentations qui nous ont été transmises par les enluminures, par les vitraux, par les sculptures et, surtout, par les sceaux ${ }^{4}$.

Les chevaliers, autant qu'ils en ont les moyens, portent le baubert, cotte de mailles couvrant le torse, les membres, le col et la tête sauf le visage. Cette défense de corps, déjà utilisée par les gallo-romains, était tombée en désuétude pendant le haut Moyen Age, sans doute à cause du recul de la sidérurgie. Avec la reprise de celle-ci, la cotte de mailles va se répandre à nouveau, pour s'imposer dés le milieu du XII ${ }^{\circ}$ siècle et jusqu'au $\mathrm{XV}^{*}$ qui voit le triomphe de l'armure de plates complètes. Toutefois, le coût élevé du haubert fait que la plupart des combattants se contentent d'un baubergeon, haubert de proportions réduites, ou même d'un simple plastron de mailles. Le casque ou beaume, dont le timbre conique du $\mathrm{XI}^{\circ}$ siècle est devenu bombé puis plat, se ferme de plus en plus et il aboutit au heaume dit de Philippe Auguste, puis au grand heaume de Saint Louis ou des Croisades. L'écu, en forme d'amande ou de triangle allongé, est de grandes dimensions, parfois légèrement courbé le long de l'axe vertical afin de mieux couvrir le corps. Il est armé, au centre, d'un umbo, bordé d'une orle et renforcé de lamelles cloutées. L'épée damassée, arme de taille dont la lame élastique, est relativement large, s'avère trop fragile contre les nouvelles défenses de corps (probablement

de hache de combat, de lance, d'épée à un seul tranchant, plutôt que de francisque, de framée et de scramasax, puisqu'on doit loyalement avouer que le sens exact de ces nots à consonnance flatteuse nous échappe» (J. HuBER'T: Bibliotbèque de l'Ecole des chartes, vol. 106, 1945-1946, pp. 140-142).

${ }^{3}$ La question a été entièrement renouvelée par l'application systématique des analyses de laboratoire: ED. SAliN: La civilisation mérovingienne d'aprés les sépultures, les textes et le laboratoire, Paris, Picard, 1949-1959, 4 vols., notamment vol. 3; Ed. Salin et A. France-Lanord: Le fer à l'époque mérovingienne, étude lechnique el archéologique, Paris, Geuthner, 1943, XVI, 291 p. (Rhin et Orient, II).

4 Dans un précédent ouvrage, Forteresses de la France médiévale, construction, attaque, défense, Paris, Picard, 1967, nous avons dit quelques mots (pp. 15-17) sur les secours que ces documents figurés peuvent apporter à l'étude de la question et sur les réserves que soulève leur emploi. Pour ce qui est des sceaux et malgré que son auteur ne se fonde que sur des exemples empruntés aux Archives nationales de Paris, les travaux essentiels demeurent: G. Demay: Le costume de guerre et d'apparat d'aprés les sceaux du Moyen Age, Paris, J. B. Dumoulin, 1875, 56 p. (extrait de Mémoires de la Société nationale des aniquatires de France, t. 35); G. DEMAY: Le costume au Moyen Age d'aprés les sceaux, Paris, D. Dumoulin, 1880, 496 p. 
à cause de ses nombreuses soudures). Elle est remplacée par une épée plus roide, plus effilée, dont la lame est faite d'une âme de fer gainée d'acier ${ }^{5}$. La lance, forte et pesante, est exclusivement une arme d'hast

FIG. 1.-Sceau d'Arthur I" de Bretayne, 1202 ( «Le corps est entièrement protégé par lic haubert. Le heaume, à nasal et à timbre bombé, laisse le visage à découvert» [G. Dimay: Le costume de guerre... d'aprés les sceaux, pl. 11$]$ ).

$$
\hat{R} \cdot 243
$$

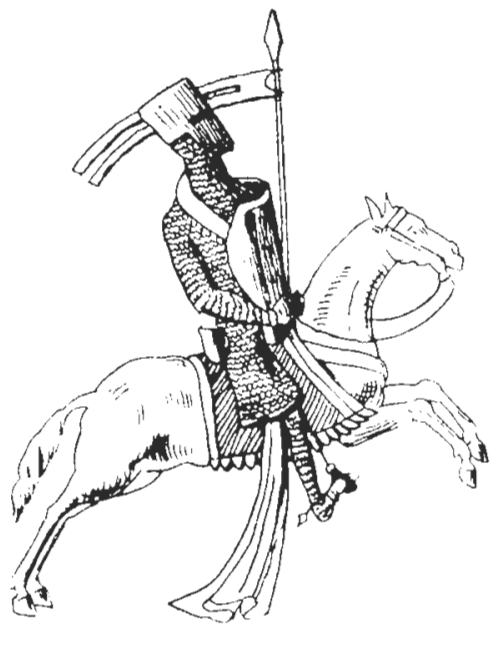

et ne peut guère être employée comme arme de jet tel que le montrait encore la broderie de Bayeux.

Les cavaliers, porteurs de l'équipement plus ou moins complet, forment l'élite des armées. Toutefois, les fantassins sont loin d'être négligeables. Il est vrai que R. Fawtier est d'avis que la valeur de ces fantassins, quasiment nulle aux XIV et $\mathrm{XV}^{\mathrm{c}}$ siècles, devait être fort contestable aux XII ${ }^{\circ}$ et XIII' ${ }^{\circ}$. Mais les travaux de J. Boussard semblent prouver que, sous les trois premiers souverains de la maison d'Anjou, les fantassins-mercenaires forment le noyau de l'armée anglaise ${ }^{7}$. Il faut également tenir compte que, si bien les guerres d'alors consistent en raids suivis de

'C. PANSERI: Richerche metallographiche sopra una spada da guerra del $X I^{\prime}$ seccolo (in: Associazione italiana di metalurgia, Milano, Documenti e contributi, quaderni 2, 1957, pp. 7-40).

"I. Boussard: Les mercenaires au XII" siècle, Henri II Plantagenêt et les origines de l'arméce de mélier (in: Bibliolbèque de l'Ecole des chartes, vol. 106, 1954-1956, pp. 189-224); F. Lot: L'art militaire el les armées au Moyen Age en Europe el dans le Proche Orient, Paris, Payot, 1947, 2 vols. (Bibliothèque historique); cf. vol. 1, pp. 217-218.

Histoire des institutions trançaises au Moyen Age, publ. sous la direction de F. Lot et de R. Fawtier, Paris, Presses Universitaires de France, 1957-1962, 3 vols.; cf. vol. 2, Institutions royales, pp. 511 et ss. 
quelques engagements en rase campagne où la cavalerie lourde joue un rôle décisif, ces actions préliminaires aboutissent normalement à l'attaque ou à la défense d'une place, la possesion de celle-ci étant l'enjeu du conflit. Dans cette guerre de sièges, les terrassiers, les ingénieurs, les charpentiers, malgré leur faible esprit militaire, sont indispensables. Quant à la lutte proprement dite, eschelades, mélées, etc., elle est le fait de combattants non montés: fantassins ou cavaliers qui ont mis pied à terre. On pourrait argumenter que, dans de nombreuses figurations, les assaillants apparaissent à cheval. Mais, outre que cette posture est, peut-être, purement conventionelle, les hommes montés, ne sont-ils pas en «position d'attente», prêts à mettre pied à terre au moment de l'action? Dans le cas du siège de Dinan (représenté dans la broderie de Bayeux), les cavaliers semblent être en train d'effectuer une charge pour s'emparer du pont d'accés; les deux guerriers qui, au centre de la scène, s'attaquent réellement à la palissade en essayant de l'incendier, sont à pied. De même,
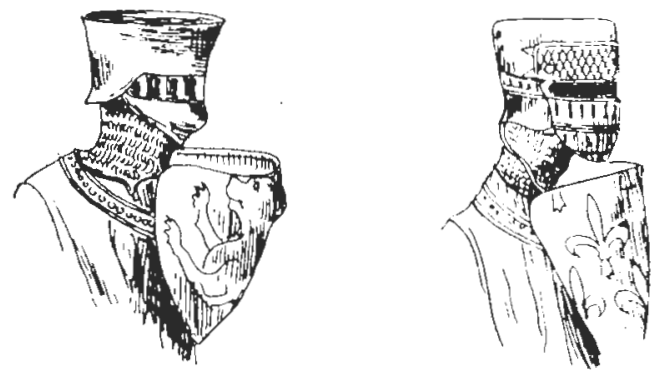

Fig. 2.-Détail des sceaux de Guillaume de Chauvigny, 1217, et du fils de Pbilippe Auguste, 1214 («Les heaumes, qui couvrent presque entièrement la tête et le visage, marquent un grand progrés sur celui représenté figure 1» [G. DEmaY: Le costume de guerre... d'aprés les sceaux, pl. [X]).

$$
\text { R. } 244
$$

Suger rapporte que, lors du siège du château du Puiset par Louis VI en 1111 , «Les chevaliers [défenseurs] qui, de toute la vitesse de leur chevaux faissaient tout le tour du château pour le défendre, accablaient de coups en survenant inopinément ceux qui s'accrochaient de leurs mains à la palissade...» ${ }^{8}$. Le cheval n'est utilisé ici que comme un moyen de transport et les défenseurs se comportent comme une infanterie montée, non point comme de véritables cavaliers. Rappelons enfin qu'à la bataille de Bouvines, les piquiers du comte de Boulogne résistent jusqu'à la fin et qu’à un certain moment, Philippe Auguste est jetté à bas de son cheval par les gens de pied et qu'il ne doit son salut qu'à la solidité de son haubert.

- Sugre: Vie de Louis VI le Gros, chap. 9, éd. H. Waquet, Paris, Champion, 1929, XXVII, 332 p. (Les classiques de l'histoite de France au Moyen Age, I1); cf. pp. 136-141. 
Que les «profesionnels» de la guerre (nobles et routiers) méprisent les simples recrues levées à l'occasion d'un conflit, cela est certain et ce mépris n'ira qu'en s'accroissant aux XIV et $X^{*}$ siècles. Que les cavaliers, à leur tour, regardent comme inférieurs ceux qui, généralement, combattent à pied, c'est probable également. Il n'y a pas si longtemps que les militaires de carrière se gaussaient des «réservistes» (bien des anciens de 14 se rappellent la phrase fameuse «cachez les 75 , voilà les réservistes») et que les cavaliers (cuirassiers, dragons, hussards) ne tarissaient pas de faire des plaisanteries sur les «biffins» et sur les «tringlots». Mais, le rôle de ces derniers était-il négligeable pour autant?

Quoiqu'il en soit, on peut dire qu'à l'époque considérée, les gents de pied, lorsqu'ils sont régulièrement armés, portent comme armes défensives: sur la tête un chapel de fer ou de cuir bouilli, de forme ronde, avec un rebord régulier, peu saillant, qu'un ourlet peut renforcer. Couvrant le torse, un gambison (vêtement rembourré d'étoupe ou de filasse) ou une cotte de cuir qui peut être tréillisée, c'est à dire, couverte de minces lanières de cuir entrecroisées. Le cas écheant, un écu similaire à celui des cavaliers mais plus léger. Comme armes de jet: l'arc, dont la force et la portée est faible, ou la fronde ou, surtout, l'arbalète. Celle-ci, parfois utilisée à l'époque gallo-romaine ${ }^{9}$, semble avoir disparu pendant le haut Moyen Age. Son emploi en 949, 984 et 985 est bien signalé par Richer ${ }^{10}$ mais celui-ci est un auteur suspect, dont les dires ne sont souvent que des amplifications litteraires. L'arbalète figure dans une enluminure du Commentarius in Ezecheliem, manuscrit datant de la fin du $\mathrm{X}^{\mathrm{c}}$ siècle ou du début du $\mathrm{XI}^{\mathrm{C}}{ }^{11}$ mais elle n'apparait pas représentée dans la broderie de Bayeux. Elle ne figure pas non plus dans le manuscrit byzantin de Scylitzes (Biblioteca Nacional de Madrid, Codex Matritensis, vit. 26-2) qui fut enluminé à la fin du XII'c siècle, début du XIII" ${ }^{12}$ et, lors de la première Croisade, Anne Comnène, fille de l'empereur Alexis, décrit soigneusement cette «arme barbare, absolument inconnue aux

"Deux cippes, déposés au musée du Puy (cote 1679 et 1683), datant du I"r ou du II" siècle de notre Ere, en donnent des représentations assez juste. Il est question des arbalètriers («arcuballistae») chez VÉGÈCE: Les institutions militaires, liv. II, chap. 15 (in: Ammien Marcellin...: Oeuvres, éd. Nisard, Paris, Didot, 1869, III, 820 p.); cf. pp. 680-681.

1" Richer: Hisioire de France, liv. II, chap. 92; liv. III, chap. 98 et 104; éd. R. Latouche, Paris, Champion, 1930-1937, 2 vols. (Les classiques de l'histoire de France au Moyen Age, 12 et 17); cf. vol. 1, pp. 282-283; vol. 2, pp. 124-127 et 132-135

"Bibliothèque nationale, Paris, latin 12.302, fol. 1.

12 A. Brunin de Horfmeyer: Military equipment in the byzantine manuscript of Scylizzes (in: Gladius, t. V, 1966, pp. 9-160); cf. pp. 137-139. 
Grecs» qu'elle voit aux mains d'un des Croisés ${ }^{13}$. L'arbalète, plus précise et plus puissante que les arcs alors en usage, se généralise en Europe dans le courant du XII ${ }^{\mathrm{e}}$ siècle, malgré la prohibition de l'Eglise (deuxième Concile de Latran, 1139) qui interdit lusage de l'arme, jugée trop meurtrière, dans les combats entre Chrétiens. Henri II d'Angleterre, son fils

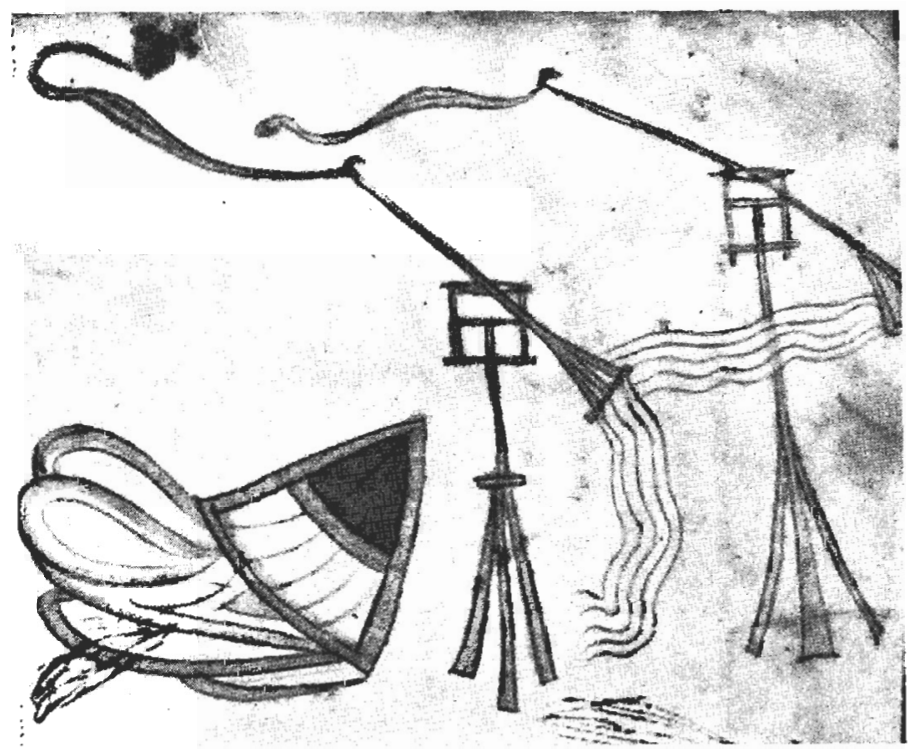

FIG. 3.-Engins à balancier, fin du XII siècle («Partie supérieure de la miniature occupant le fol. 114 du manuscrit de Petrus de Eboli. On distingue nettement la fronde à projectiles ainsi que les liens servant à entrainer la verge de l'engin» [Cl. Bibliothèque de la Bourgeoisie, Berne]).

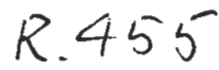

Richard Coeur de Lion et Philippe Auguste, organisent des corps d'arbalétriers à pied ou à cheval, s'en servent courament et les flèchiers (ou archers) disparaissent presque totalement des armées du XIII ${ }^{e}$ siècle.

Les autres armes offensives du piéton peuvent être fort diverses. Les faucharls, les guisarmes, les vouges, etc., consistent fondamentalement en une hampe armée d'un large fer propre à servir d'arme de taille et à

13 Anne. Comnène: Alexiade, liv. II, chap. 8, éd. B. Leib, Paris, Les Belles Lettres, 1937, 3 vols. (Coll. byzantinc... G. Budé, 8, 11, 12); cf. vol. 2, pp. 217-218. 
trancher les jarrets des chevaux, et terminé en pointe pour frapper d'estoc; un ou plusieurs crochets, fixés prés du fer, servent à désarçonner les cavalicrs. L'épée ou la bache danoise (hache à long manche, maniée des deux mains), parfois la massue ou l'épieu complètent la panoplie.

Les machines de jet sont, essentiellement, des engins à balancier, du type dit «à traction humaine», tels qu'ils apparaissent représentés dans le manuscrit de Petrus de Eboli, exécuté à l'extrème fin du XII ${ }^{c}$ siècle ${ }^{14}$

I.lG. 4.--Engin à balancier, débul du $X I I I^{\circ}$ siècle ("Partie inférieurc du bas-relicf existant à la cathédrale de Carcassonne et qui est.présumé avoir orné le tombcau de Simon de Montfort. En haut, à droite, Ie projectile est placé dans la fronde. En bas, à gatuche, l'engingneor. $A$ droite et au centre, les femmes qui halent l'engin. L'extrémité de la branche courte de la verge parait garnie d'une ébauche de contrepoidss [E. V1OLLETLE-DUC: Dict. de l'archilect, vol. 8, page 389]).

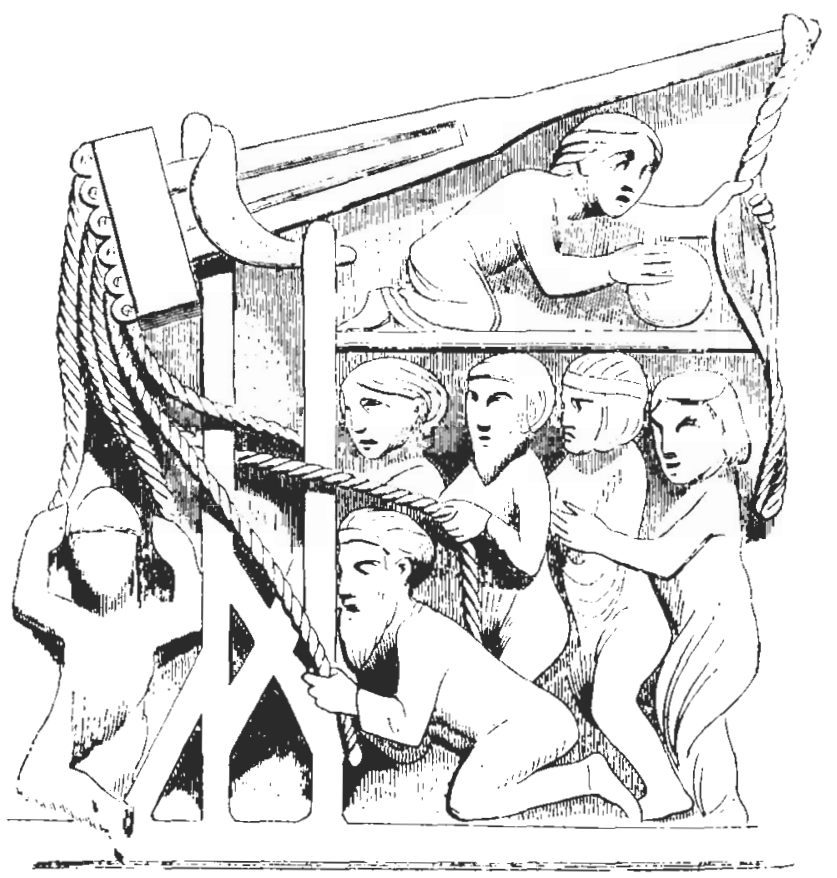

ou dans le bas-relief de la cathédrale de Carcassonne (début du XIII"). Les poids du boulet lancé par ces engins devait être de l'ordre de $50 \mathrm{~kg}$. Il est vrai que Guillaume le Breton, dans son récit du siège du château de Boves par les troupes de Philippe Auguste parle d'engins lançant de

14 Bibliothèque de la Bourgeoisie, Berne, ms, 120, exemplaire en partie autographe. Ce manuscrit a fait l'objet de diverses reproductions facsimilaires, p. e.: PÍ́tro bl: Enoli: Liber ad bonorem Augusti, éd. G. B. Siracusa, Roma, Istituto Storico Italiano, 1905-1906, 2 vols.; Petri Ausol.rni de Ebulo: De rebus siculis carmen, éd. E. Rota, Città del Castello, S. Lapi, 1904. 
véritables quartiers de roc ${ }^{15}$. Mais le chroniqueur exagère probablement et on ne saurait voir dans sa phrase autre chose qu'une marque d'admiration; tout au plus, une indication du nombre d'hommes requis pour la manoeuvre. Toutefois, la puissance des projectiles devait être assez considérable puisque Guillaume le Breton dit qu'ils démolissent des hourdages, des créneaux et même des remparts.

Les frondes à manche sont des armes plus légères. Appelées fustibales par les gallo-romains ${ }^{16}$, elles seront encore utilisées au $\mathrm{XV}^{\mathrm{c}}$ siècle, comme le prouve la mention des «16 frondes à bâton» adquises lors du siège d'Orléans ${ }^{17}$.

Ces engins peuvent être construits d'avance et entreposés dans les places royales d'où on les transporte, plus ou moins démontés, sur les lieux de l'action. Il en est de même des diverses armes. Elles sont accumulées dans ces véritables arsenaux et quelques inventaires nous en sont parvenus ${ }^{18}$. Quant à leur fabrication, les comptes des baillis et des prévôts royaux pour 1202, nous transmetent diverses précisions. Ainsi, p. e., on y trouve mentionné le nom de Geoffroi de Montfort, qui reçoit une solde annuelle de 36 livres et auquel diverses sommes sont allouées pour les cordes et autres fournitures; on y trouve également les noms de Renier, de maître Raymond, de maître Bernard, armuriers eux-aussi, ceux de fabricants de heaumes, etc. ${ }^{19}$.

Les noms de quelques «artilleurs», sont connus également. Les balistaires Jourdain, Renaud Tatin, Paviot, etc., se distinguent au siège du Château-Gaillard et ils reçoivent des terres, probablement en recompense de leurs services. Les deux premiers devaient être assez riches puisque,

15 Rigord et Gulllaume le Breton: Ocuvtes, liv. II, éd. H. F. Delaborde, Paris, Renouard, 1882-1885, 2 vols. (Société de l'Histoire de France); cf. vol. 2, p. 54. Le texte de Guillaume le Breton est le suivant: «... molares // incircumcisos et magni ponderis, ut vix // tollatur manibus bis quator unus eorum.»

16 Modestus: Précis des termes de la milice, $\$ 12$ (in: Ammien Marcellin...: Oenures, éd. Nisard, Paris, Didot, 1869, III, 820 p.); cf. p. 647.

${ }_{17}$ Joumal du siège d'Orléans, éd. P. Charpentier et Ch. Cuisard, Orléans, H. Herluison, 1896, LVI, 410 p.; cf. p. 321.

"Recueil dit Cartulaire A de Philippe Auguste (Bibliothèque Vaticane, ms. Ottoboni n." 2796), reproduit, facsimilairement sous le titre Le premier registre de Philippe Auguste, éd. L. Delisle, Paris, H. Champion, 1883, 20 p. et pl. Inventaires publiés in: Ed. Audon: Essai sur l'armée royale au temps de Philippe Auguste, nouv. éd., Paris, H. Champion, 1913, 234 p.; cf. pp. 187-197.

"Les comptes de 1202 sont publiés in: N. Brussel: Nouvel examen de l'usage général des fiefs, $2^{\circ}$ éd., Paris, J. de Nully, 1750, 2 vols.; cf. vol. 2, pp. CXXXIX-CC. La transcription faite par Brussel a été reproduite facsimilairement et pourvue d'un index analytique, in: F. LOT et R. FAwTIER: Le premier budget de la monarcbic française, le compte général de 1202-1203, Paris, Champion, 1932, 300, CXXXIXCC p. (Bibliothèque de l'Ecole des hautes études, Sec. Histoire et philologie, n." 259). 
en 1227 , ils se portent caution - pour des sommes relativement considérables - de la bonne conduite de Cadoc, emprisonné par ordre de Philippe Auguste pour diverses exactions et que la reine Blanche de Castille faisait mettre en liberté à la mort du roi ${ }^{20}$.

Notons enfin que la ruine des ouvrages attaqués peut être obtenu au moyen de travaux de mine ou de sape et de nombreux exemples pourraient être allegués dans ce sens ${ }^{21}$.

\section{LES EFFECTIFS}

Les travaux d'Audoin ${ }^{22}$, ceux de F. Lot ${ }^{23}$ et les remarques de R. Fawtier ${ }^{24}$ qui ont tempéré les conclusions parfois trop tranchantes de Lot, permettent de ramener à de justes proportions les chiffres avancés par les chroniqueurs. La coutume, corrigeant le principe féodal qui veut que le vassal aide son suzerain «de toutes ses forces», fait que le vassal n'apporte qu'une partie des troupes que lui-même éxigerait pour son service. Ainsi, le duc de Normandie, qui peut requérir l'aide de 581 chevaliers, n'en présente au roi que le tiers; la Bretagne, qui dispose de 166 chevaliers, en fournit 40 , soit à peu prés le quart; le Ponthieu 16 au lieu de 60 , etc. ${ }^{25}$. L'obtention de ce concours, si faible soit-il, est toujours aléatoire et donne lieu souvent à de véritables négociations diplomatiques. En outre, le service féodal est de courte durée, quarante jours en moyenne. Pour tenir campagne plus longtemps, il faut rétribuer ceux qui, ce délai écoulé, acceptent de rester. La seule ressource, pour avoir des hommes toujours disponibles, consiste à embaucher des mercenaires. Ces troupes gagées, qui ont du exister de tout temps, si bien en proportion variable prénent une importance croissante ${ }^{26}$. En Angleterre, dés Henri $\mathrm{I}^{\mathrm{cr}}$, on trouve un impôt spécial, l'écuage ou sculagium, rachat du service personnel, qui permet de les solder. Henri II lève l'écuage jusqu'à sept fois pour se procurer des merccnaircs et fait de ceux-ci le noyau de ses armées. Ces hommes, pour la plupart recrutés dans les provinces du Nord de la France - en particulier le Brébant, d'où leur nom de brabançons ou de cottereaux — sont de véritables profesionnels. Bien entraînés,

20 Rigord et Guillaume le Breton: Oeuvres, éd. H. F. Delaborde, vol. 2, pp. 186-187 et notes.

${ }^{21}$ Ihid., vol. 1, pp. 94-95 et 115; vol. 2, pp. 53, 203-204 et 207.

22 Ed. Audoin: Essai sur l'arméc royale an temps de Pbilippe Auguste.

${ }_{23}$ F. Lot: L'art militaire et les armées au Moyen Age.

${ }^{24}$ Histoire des institutions françaises, vol. 2, pp. $511-535$.

${ }^{25}$ F. Lot: Liart militaire et les armées au Moyen Age, vol. 1, p. 219.

${ }^{26} \mathrm{~J}$. Boussard: Henri II Plantagenôt et les origines de l'armée de métior, pp. 192-195. 
bien équupés (en partie aux frais du roi), conduits par des chefs tels Mercadier ${ }^{27}$, ils se distinguent dans la plupart des actions de guerre et Philippe Auguste, quoique en moindres proportions, emploie également de routiers ou soudoyers, dont Cadoc est le chef le plus célèbre.

Ceci étant, l'armée dont dispose Philippe Auguste est peu nombreuse. Des renseignements précis nous sont transmis par la Prisia Servientum ou Prisée des Sergents ${ }^{28}$. Les sergents sont les agents du roi dans ses terres, levés par prévôtés, par abbayes, par communes. Ils doivent servir trois mois chacun, mais le roi peut, à son gré, remplacer ce service par un versement de 3 livres parisis, fait par la commune. En 1204, le nombre de ces sergents oscile entre 7.695 et 8.054 . L'armée royale permanente compte environ 3.043 hommes ainsi répartis: 257 chevaliers, 267 sergents à cheval, 86 arbalètriers à chéval, 133 arbalètriers à pied ${ }^{29}$, 2.000 sergents à pied, 300 routiers. Il faut y ajouter, en temps de guerre, les contingents féodaux qui, en 1216, semblent avoir été de l'ordre de 800 chevaliers $^{30}$.

Les armées vivent, principalement sur le pays, soit-il ami ou ennemi. Toutefois, chaque seigneur, ville, prévôtée ou abbaye, fait suivre son contingent d'un ou plusieurs chariots portant les impedimenta, et la fourniture de charrettes est prévues expressément dans la Prisia Servientum. Pour les expéditions de longue durée, on accumule vivres et équipements dans certaines places servant de bases d'opération. Telles sont Bourges et Mantes pour Philippe Auguste, Bordeaux, Chinon, Nantes, Poitiers, Rouen, etc., pour les Plantagenêts et c'est probablement l'éloignement de sa base de Cahors qui pousse Henri II, comme plus tard son fils Richard, à saisir un prétexte pour reculer devant Toulouse ${ }^{31}$.

\section{LES FORTERESSES}

Les guerres d'alors consistant surtout dans l'attaque et la défense des places, tout ce qui se rapporte à la fortification revêt une importance capitale. Les Plantagenêts furent de grands bâtisseurs de forteresses. Phi-

${ }^{27} \mathrm{H}$. GÉrnud: Mercadier, les routiers an XIII siècle (in: Bibliothèque de l'Ecole des chartes, vol. 3, 1841-1842, pp. 417-443).

${ }^{2 *}$ Ce document figure au f. $91^{*}$ du Cartulaire A de Philippe Auguste. Publié, ainsi que le texte d'une traduction du XIV' siècle, in: ED. AudoIN: Essai sur l'amée royale, pp. 123-135.

2) Les arbalc̀triers à pied, moins coûteux que ceux à cheval, les dépassent largement en nombre. Une échelle des soldes des chevaliers, des arbalètriers, des sergents, etc., est fournie in: ED. Audoin: Essai sur l'armée royale, pp. 113-114.

3" F. Lot: L'art militaire et les armées au Moyen Age, vol. 1, pp. 219-220.

3i J. Boussard: Henri II Plantagenét et les origines de l'armée de métier, p. 222. 
lippe Auguste en fit de même et malgré qu'un certain nombre de ses constructions militaires aient disparu aujourd'hui, il en subsiste un ensemble considérable ${ }^{32}$. Ces édifices, dont la plupart ont été étudiés à plusieurs reprises ${ }^{33}$, peuvent être rangés sous deux chefs principaux: les ouvrages bâtis de toutes pièces ou rénovés de fond en comble et les additions faites à une forteresse préexistante pour en renforcer la défense ou pour l'adapter à un rôle nouveau.

Pour ce qui est du premier groupe - Dourdan, l'enceinte et le Louvre de Paris, Rouen, Yèvre-le-Châtel, etc. - les aspects communs peuvent être résumés ainsi: plan régulier, généralement rectangulaire (Louvre, Dourdan), parfois en losange (Yèvre-le-Châtel) ou polygonal (Rouen). Parements lises et uniformes. Courtines talutées, épaises d'environ $2,50 \mathrm{~m}$. à $3 \mathrm{~m}$., et hautes d'une douzaine. Angles armés de tours cylindriques, à base pleine. Défense du pied des murs assurée par des hourds de bois. Archères des étages successifs disposés en quinconce, pour mieux distribuer les champs de tir et ne pas afaiblir la maçonnerie le long d'une même verticale. Porte encadrée par deux tours cylindriques (à Yèvre-le-Châtel, les baies étant percées à gauche de la tour la plus proche, l'assaillant peut se couvrir de son bouclier). Donjon remplacé par une maitresse-tour placée à un angle de l'enceinte et pouvant communiquer directement avec la campagne (le cas du Louvre, qui fait exception, sera considéré plus loin).

La modernisation d'une forteresse déjà existante, peut donner lieu à des travaux fort divers. Ainsi, à Gisors, il semble bien que l'on puisse attribuer à Philippe Auguste le surélèvement de la courtine fermant la

32 A. DE Dion: Note sur les progrés de l'architecture militaire sous le règne de Pbilippe Auguste (in: Mémoires et documents publiés par la Société d'archéologie de Rambouillet, t. 1, 1870-1871, pp. 157-181); tiré à part: Rambouillet, imp. de Raynal, 1871, 25 p. Trés sensément, l'auteur ne sépare pas l'ćtude de l'architecture militaire de celui de la poliorcétique contemporaine: P. HÉLIOT: La génèse des chateaux de plan rectangulaire en France et en Angleterre (in: Bulletin de la Société nationale des antiquaires de France, 1965, pp. 238-257); Les châteaux forts en France du $X^{\prime}$ au XII" siècle à la lumière de iravaux récents (in: Joumal des savants, 1965, pp. 483-514); Le Chateau-Gaillard et les forteresses des XII et XIII siècles en Europe occidentale (in: Châteat-Gaillard, études de castellologie européenne, vol. 1, Caen, 1964, pp. 53-75); L'âge du château de Carcassonne (in: Annales du Midi, 1966, pp. 7-21); J. Vallery-Radot: Quelques donjons de Pbilippe Auguste (in: Bulletin de la Société nationale des antiquaires de France, 1964, pp. 155-160). Comme ouvrages généraux, récents, voir notamment: Fr. GébéLin: Les châteaux de France, Paris, Presses Universitaires de France, 1962, 184 p.; cf. pp. 39-50; R. RitTer: Châteaux, donjons et places-fortes; l'architecture militaire française, Paris, Larousse, 1953, 209 p.; cf. pp. 52-80.

${ }_{33}$ Pour alléger les références, nous nous contentons de renvoyer à la bibliographie insérée dans nos Forteresses de la France médiévale, pp. 461-474 et addenda. 
barbacane au nord ainsi que certaines additions à l'ensemble formé par la porte et la tour du Gouverneur. A Caen, le donjon normand est entouré d'une forte chemise rectangulaire, cantonnée de tours cylindriques. Toutefois, dans la plupart des cas, on renforce le bâtiment primitif par une grosse tour et un grands nombre d'exemples de cette solution peuvent

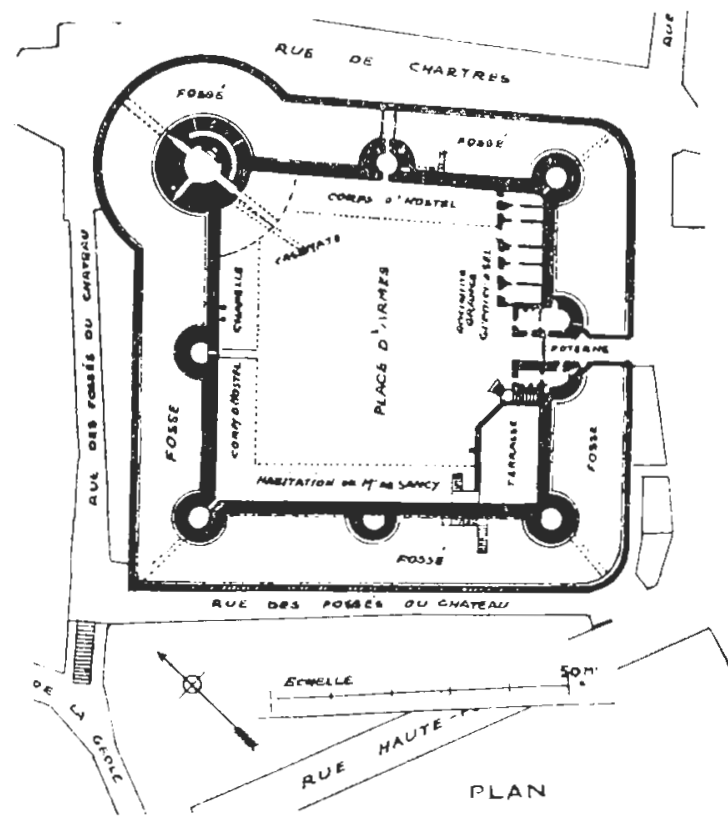

FIG. 5.-Plan du château de Dourdan ( «Ce château, bâti vers 1220 , est un des meilleur exemples de "la formule de Philippe Auguste" qui nous soient parvenus. Il est situé à l'extrémité de la ville et, primitivement, la grosse leur exemples de "la formuse à l'angle nord, était séparé du reste du château, l'enccinte de celui-ci s'échancrant en arc de cercle pour lui laisser place. Au XVI" siècle, Sully fit combler la branche intérieure du fossé, abattre le pan de muraille - indiqué par une suite de tirets- et, en prolongeant les courtines latérales du château, il intégra le donjon à l'enceinte» [G. Tessifr: Le châleau de Dourdan, page 33]).

être cités: Bourges, Chinon, Falaise, Gisors, Lillebonne, Orléans, Péronne, Verneuil, Villeneuve-sur-Yonne, etc. Notons, en passant, que pendant longtemps il en sera fait de même. Ainsi, en 1253, Alphonse de Poitiers modernise le château de Najac et fait construire, entre autres ouvrages, une grosse tour cylindrique, trés similaire d'aspect à celles bâties par Philippe Auguste, et servant de nouveau donjon. Au XVc siècle, à Loches, on ajoute la tour Neuve à l'ensemble d'ouvrages bâtis à diverses époques autours du vieux donjon rectangulaire.

Ces grosses tours présentent une série de caractères comuns: Plan circulaire. Deux des étages, au moins, sont voûtés d'ogives généralement à six branches, dont les nervures retombent sur des culs-de-lampe. «Lorque ces culs-de-lampe sont ornés de feuillages, leur décor est celui du 


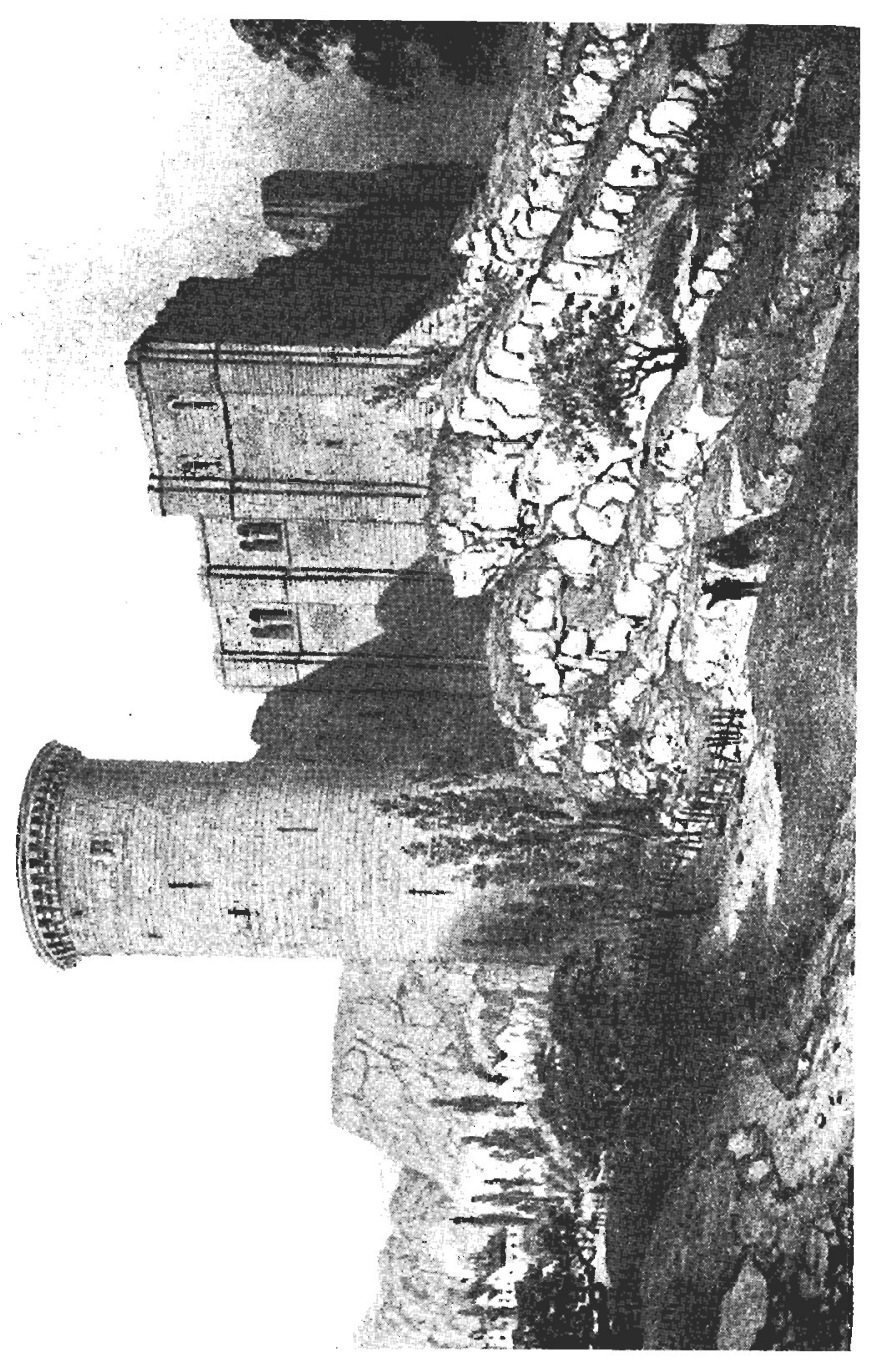

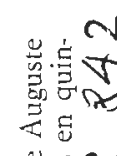

总

를

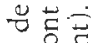

$\exists$ i

용

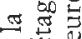

㟧.

要况奇

ธิ

घ웜

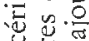

总志

政

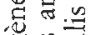

in

베니

च $\bar{g}$

ह

동

要需

出

过䍃

छ

8.

跣-

总范

就

过

50

녹

1

b.

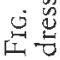


style gothique de l'Ile-de-France. Il en est de même de la modélature» ${ }^{34}$ Entrée située habituellement au niveau du rez-de-chaussée (Dourdan, Laon, Louvre, Rouen, Verneuil, Villeneuve-sur-Yonne); lorsque l'entrée est placée à l'étage supérieur, c'est pour pouvoir communiquer directement avec le chemin de ronde des courtines (Chinon, Gisors). Aménagements intérieurs assez poussés: cheminées (souvent pourvues d'un four), puits, latrines. Socle taluté mais absence d'éperon, c'est à dire du massif de maçonnerie destiné à renforcer le secteur le plus exposé au tír ennemi et à mieux étaler les projectiles lancés depuis les défenses supérieures. Le cas de la tour Blanche d'Issoudun, armée d'un puissant éperon, est exceptionnel; il s'explique probablement «par sa date [précoce] et par les traditions régionales qu'elle devait à sa situation topographique» ${ }^{35}$.

QUELQUES GROSSES T'OURS DE PHILIPPE AUGUSTE

\begin{tabular}{|c|c|c|c|c|c|}
\hline & Dale & Hauteur & $\begin{array}{l}\text { Diamètre } \\
\text { bors-oeuvre }\end{array}$ & \begin{tabular}{|c|} 
Epaisseur \\
des murs
\end{tabular} & $\begin{array}{l}\text { Etages } \\
\text { voûlés }\end{array}$ \\
\hline$\because$ Bourges $\ldots . . . . . . . . . . . .$. & $1189-1190$ & $38 \mathrm{~m} . ?$ & $20 \mathrm{~m} . ?$ & & 3 \\
\hline Chinon............... & post. 1204 & $22 \mathrm{~m}$ & $12 \mathrm{~m}$. & $3,35 \mathrm{~m}$ & 2 \\
\hline Dourdan ................ & 1222 & $26 \mathrm{~m} . ?$ & $13,50 \mathrm{~m}$ & $3,75 \mathrm{~m}$ & $2 ?$ \\
\hline Falaise & 1207 & $35 \mathrm{~m}$ & & $4 \mathrm{~m}$. & 3 \\
\hline Gisors $\quad . . \ldots \ldots \ldots \ldots$ & $?$ & $28 \mathrm{~m}$ & $13,80 \mathrm{~m}$ & $3,90 \mathrm{~m}$ & 3 \\
\hline Issoudun $\ldots$ & 1202 & $27 \mathrm{~m}$. & $15 \mathrm{~m}$. & & 1 \\
\hline * Laon. & $1204-1212$ & $29 \mathrm{~m} . ?$ & $18,48 \mathrm{~m}$. & $4,95 \mathrm{~m}$ & 2 \\
\hline * Louvre ... & $1202 ?$ & $40 \mathrm{~m} . ?$ & $20 \mathrm{~m}$ & $4 \mathrm{~m}$ ? & $?$ \\
\hline Lillebonne & post. 1214 & $28 \mathrm{~m}$. & $16,70 \mathrm{~m}$ & $4,25 \mathrm{~m}$ & 3 \\
\hline *Orléans & $1204-1212$ & $27 \mathrm{~m}$ & $16,50 \mathrm{~m}$ & $4,95 \mathrm{~m}$ & $2 ?$ \\
\hline * Péronne & $1204-1212$ & $27 \mathrm{~m}$ & $17,16 \mathrm{~m}$ & $4,62 \mathrm{~m}$ & $2 ?$ \\
\hline Rouen & 1205 & $30 \mathrm{~m}$ & $15 \mathrm{~m}$. & $4 \mathrm{~m}$ & 2 \\
\hline Verneuil & $?$ & $35 \mathrm{~m}$. & $16 \mathrm{~m}$ & $3,85 \dot{\mathrm{m}}$ & \\
\hline Villeneuve-sur-Yonne.. & $1204-1212$ & $27,28 \mathrm{~m}$. & $16,50 \mathrm{~m}$ & $4,95 \mathrm{~m}$ & 2 \\
\hline
\end{tabular}

Les édifices marqués d'un * ont disparu aujourd'hui.

${ }^{34}$ J. Vallery-Radot: La tour Blanche d'Issoudun (in: Château-Gaillard, vol. 1, 1964, pp. 150-160); cf. p. 157.

35. Ibid., pp. 159-160. 
Un tableau, établi d'aprés les travaux de Vallery-Radot, réunit quelques données relatives à ces tours.

L'ampleur du programme de construction requis par l'extension et la durée du conflit, pousse à la création, chez les deux adversaires, d'une sorte de service central. La formation progressive de celui-ci autour du trône des Plantagenêts semble apparaître dans les documents anglais ${ }^{36}$, et Philippe Auguste dut en faire de même. Naturellement, il ne saurait être question d'un véritable service, avec tout ce que ce mot évoque pour nous aujourd'hui. L'organisation administrative, encore incipiente chez le Capétien, rendrait une telle idée impensable. Il devait s'agir, simplement, d'un certain nombre d'hommes du métier, plus ou moins attachés à la personne du roi. Leur formation devait être toute pratique: connaissances transmises par voie orale, de maître à élève, et enseignement direct sur le chantier, sur le tas. Cependant, l'existence de «livres de chantier» ou de cahiers de notes n'est pas exclue. L'Album, de Villard de Honnecourt, qui date du XIII siècle, est un exemple célèbre de ces sortes de formulaires de praticiens ${ }^{37}$. D'autres ont pû exister et il y a longtemps déjà que $\mathrm{V}$. Mortet a relevé deux chapitres relatifs aux fondations des édifices et des ponts, contenus dans un formulaire du VIII ${ }^{\circ}$ siècle, le Mappae clavicula de efficiendo auro ${ }^{38}$.

C'est dans ce milieu qu'a dû être élaborée «la formule de Philippe Auguste», pour reprendre l'heureuse expression de Fr. Gébelin ${ }^{39}$. Mais, comment est-elle née? Gébelin a mis en relief les ressemblances que présentent les constructions de Philippe Auguste avec celles des architectes gallo-romains. Ces ressenblances s'expliqueraient par la fréquentation des auteurs de l'Antiquité (tels Végèce), par l'examen des exemples offerts par les murailles gallo-romaines (p. e., Senlis) et par celui des réalisations byzantines (Byzance fut l'héritier direct de Rome) que le roi avait pu voir lors de la troisième Croisade ${ }^{40}$. Ces observations sont fort pertinentes. Ainsi, au moment où Philippe Auguste réalise ses grands travaux, l'Université de Paris existe depuis plus d'un demi siècle et des clercs tels frère Guérin - ancien Hospitalier - comptent parmis les conseiller du roi. Or, Végèce était répandu dans le monde des clercs et,

"P. Hél1ot: Le Château-Gaillard, p. 68.

${ }^{37}$ L'Album de Villard de Honnecourt (Bibliothèque nationale, ms. français 19.093) a fait l'objet de plusieurs reproductions facsimilaires. Citons, notament, l'édition de H. R. Hahnloser, Wien, A. Schroll, 1935, réimprimée à Graz, Akademische Druck u. Verlag, 1966, et celle de Th. Bowie, Blomington, Indiana University Prcss, 1959.

"V. Mortet: Un formulaire du VIII" siècle (in: Bulletin monumental, vol. 71, 1907, pp. 442-465).

$30^{\circ}$ Fr. Gébelin: Les cbâleaux de France, p. 39.

to Ibill, pp. 43-45. 
jusqu'à un certain point, dans celui des laics lisant le latin ${ }^{41}$. Cependant, il ne faut pas oublier que l'architecture étant un art de combinaisons géométriques et que les techniques étant similaires chez les gallo-romains et chez les contemporains de Philippe Auguste, certaines coincidences ont pû se produire sans qu'il y ait eu de copie proprement dite ${ }^{42}$. De même, la reprise de certains moyens d'attaque entraine la résurrection des défenses correspondantes. Tel est le cas des travaux de mine et de sape. Pratiqués par les Romains et par les peuples de l'Orient classique, ils étaient inusités des Barbares qui envahissent l'Empire et les constructeurs galloromains purent n'en pas tenir compte dans leurs ouvrages. Leur emploi s'étant répandu à nouveau au temps de Philippe Auguste, il faut pousser en profondeur les fondations des édifices militaires, en renforcer le socle par de larges talus et renoncer à la motte, afin d'asseoir directement les donjons sur le sous-sol rocheux. Dans certains ouvrages, comme c'est le cas à Yèvre-le-Châtel, un arc noyé dans la maçonnerie des courtines est bandé entre les socles des tours latérales; cet artifice, déjà connu des Byzantins au $\mathrm{VI}^{\mathrm{e}}$ siècle, rend aléatoire les travaux du pionnier. Il est donc fort probable que les constructeurs de Philippe Auguste ont élaboré une doctrine propre (aujourd'hui on est trop enclin à refuser à l'homme médiéval la subtilité et la réflexion que l'on accorde volontier au sauvage le plus primitif...), doctrine qui devait tenir compte du but assigné aux nouvelles constructions ainsi que des circonstances où elles étaient nées.

Ces forteresses sont exclusivement militaires. Elles ne sont pas destinées à servir d'habitation à un seigneur et aux siens, mais à un officier chargé par le roi de gouverner la place. Cet officier doit pouvoir se rendre, inmédiatement, là où sa présence est nécessaire. En cas de conflit, ce n'est pas un seigneur isolé, assiègé par un autre seigneur, qui n'ayant nul secour extérieur à attendre, se retranche dans son donjon. La mission de l'officier royal est toute autre. Il doit, naturellement, se défendre le plus longtemps possible, mais il doit aussi advertir le roi qui, le cas écheant, pourra lui porter secours. Par conséquent, il ne se barricade pas dans un donjonréduit, d'accés difficile, dont la porte s'ouvre au niveau du premier étage et dont l'entrée est barré par une série de chicanes. La grosse tour où il se tient est placée à un endroit tel qu'il puisse surveiller et rayonner en toutes directions et si le château est bâti jouxte l'enceinte d'une ville, la

"Une étude à propos de la diffusion de Végèce au Moyen Age serait fort souhaitable.

${ }^{42} \mathrm{~A}$ propos des outils, des arts du bâtiment et des techniques médiévales, outre les Dictionnaires de Viollet-le-Duc, voir notamment: B. GiLlE: Le Moyen Age en Occident, $V^{e}$ siècle-1350 (in: Histoire générale des techniques, vol. 1, Paris, Presses Universitaires de France, 1962); cf. pp. 427-598; A History of technology, vol. 2, Oxford, Clarendon Presse, 1957, passim. 
tour se dressera à l'opposite de cette enceinte. Le donjon du Louvre fait exception, mais son emplacement au milieu de la cour centrale s'explique peut-être par le rôle symbolique qui lui est attribué (tous les donjons féodaux mouvant de celui du Louvre); en outre, c'est là que furent déposées les archives royales reconstruites aprés la déroute de Fréteval. L'entrée au niveau du rez-de-chaussée, facilite les déplacements et une porte suplémentaire permet des communications aisées avec l'extérieur de la place.

D'autre part, l'ensemble de ces travaux ayant été exécuté en une quinzaine d'années, en plein conflict, il est naturel que l'on se soit efforcé de simplifier la tâche. D'où adoption d'une sorte de «plan-type» et choix des éléments compte tenu de l'efficacité militaire, de la facilité d'exécution et de la réduction du travail. L'usage constant des tours et des donjons cylindriques en est un exemple. Le tracé circulaire est, militairement, supérieur aux tracés carrés ou rectangulaires. Les théoriciens antiques le recommandent. Les enceintes gallo-romaines en offrent de nombreux modèles. Sa réalisation sur le terrain est plus aisée que celle des donjons aux formes complexes tels Houdan, Etampes ou Provins. Autant de raisons qui poussent à son adoption systématique.

Ce choix délibéré, qui parait s'accorder avec ce que l'on sait de la personne de Philippe Auguste, porte à attribuer à celui-ci une action décisive dans l'élaboration de la doctrine et l'empreinte du Capétien serait plus marquée dans ses constructions que celle des Plantagenêts dans les leurs ${ }^{43}$.

Des devis de construction, fort précis, sont contenus dans le recueil dit Cartulaire $A$ de Pbilippe Auguste ${ }^{44}$. Ils concernant les travaux de fortification à exécuter dans des villes telles Paris, Corbeil, Melun, Montargis, ainsi que la construction de donjons à Orléans, à «Villenove» ${ }^{45}$, à Laon, à Péronne, à Cappy, à Ribemont, etc. Voici, à titre d'exemple, celui relatif à Orléans:

«Tascia Aureliani. Turris debet habere XIIII tesias de alto et murus $\mathrm{XV}$ pedes de spisso et XX pedes de concavo, fossatum XL pedes latitu-

${ }^{43}$ P. HÉLiot: Le Cháleau-Gaillard, p. 69.

44 Voir ci-dessus note 18 . Un choix de textes concernant les travaux de constructions de Philippe Auguste est donnée in: A. Tuetey: Rapport sur une mission à Rome relative au cartulaire de Philippe Auguste (in: Archives des missions scientifiques et littéraires, $2^{*}$ série, t. 6, 1880); cf. p. 350; V. Mortet et P. Descham ps: Recueil de textes relatits à l'bistoire de l'arcbitecture... [XI'-XIII' siècles], Paris, Picard, 1911-1929, 2 vols.; cf. vol. 2, pp. 214-220; J. VALLERY-RADOT: Quelques donjons de Pbilippe Auguste, pp. 156-157.

${ }^{45}$ «Villenove» n'est autre que Villeneuve-sur-Yonne. Cf.: ED. Audorn: Essai sur l'armée royale, p. 196, $\$ 28$ et note h; A. DE CaUmont: Abécédaire... archilecture civile et militaire, $2^{e}$ éd., Rouen, Hardel, 1858, p. 418; J. Vallery-Radot: Quelques donjons de Philippe Auguste, pp. 158-159. 
dinis et pavatum de foris et XX pedes de profundo, et duo pontes tornatiles, et hordamentum totum paratum de ferro et ligno, et de omnibus istis debet habere magister Guillelmus XIIII" libras.»

A la mention de «magister Guillelmus» prés, un libellé analogue est utilisé pour Villeneuve et pour Laon, les côuts et les dimensiones seuls varient; pour Péronne, la rédaction change quelque peu, mais le fond reste analogue. Les dimensions de la tour, celles du fossé «revêtu», la double porte, etc., sont toujours clairement spécifiées. L'examen de la tour de Villeneuve (seule qui subsiste aujourd'hui, les trois autres ayant disparu) montre que les constructions s'ajustaient exactement aux devis; autrement dit, que l'on bâtissait conformément à un modèle-type, qui parait être le donjon du Louvre; à propos de la tour de Dun (Dun-le-Roi, aujourd'hui Dun-sur-Auron) il est noté «expensas de domo Duni... pro faciendo turri, et de ballio faciendo, ad mensuram turris Parisius, XIII C $1 .{ }^{40}$.

Dans les textes on relève également les noms de divers maîtresmaçons qui apparaissent comme responsables de l'exécution des travaux. Tels sont maître Eudes, maître Gautier, maître Garnier, maître Guillaume de Flamenville, etc., mais P. Héliot a fait remarquer qu'il semble qu'on eut affaire ici, moins à de véritables ingénieurs militaires travaillant d'aprés leurs propres idées, qu'à des personnes qualifiées, chargées de veiller à ce que les prescriptions royales fussent dûment exécutées ${ }^{47}$.

La «formule de Philippe Auguste» est à la base de presque toutes les constructions défensives bâties en France en XIII ${ }^{e}$ siècle. Coucy, Montaiguillon, le Coudray-Salbart, Boulogne-sur-Mer, La Fère-en-Tardennois, Angers, et bien d'autres encore, dérivent, tous, du modèle capétien et, à leur tour, ils en inspirent d'autres. Ainsi, lorqu'en 1282, Charles I ${ }^{\text {cr }}$ d'Anjou décide de construire le Château Neuf de Naples, l'architecte Pierre d'Agincourt le bâti à l'image de celui d'Angers.

4h N. Brussel: Nouvel examen, vol. 2, p. CLIII. Voir ci-dessus note 19.

${ }^{47}$ P. Hélıo': Le Château-Gaillard, pp. 68-69. 